\title{
OPEN Increased red cell distribution width predicts severity of drug-induced liver injury: a retrospective study
}

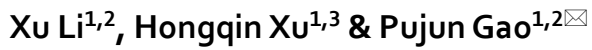

We used a retrospective study design to evaluated the predictive value of red cell distribution width (RDW) for drug-induced liver injury (DILI) severity in Chinese patients with liver biopsy to assist with early DILI management. We included 164 DILI patients with complete laboratory information and medical history. We compared outcomes of 36 patients with severe DILI with outcomes of a control group of 128 patients with mild-to-moderate DILI. Multivariate analyses of risk factors for severe liver injury in Chinese patients with DILI revealed an estimated adjusted odds ratio (AOR) $(95 \% \mathrm{Cl}$ ) of 4.938 (1.088-22.412) in patients with drinking. Risk for serious liver injury was also increased significantly in patients with dyslipidemia [AOR $(95 \% \mathrm{Cl}) 3.926(1.282-12.026)]$, higher serum total bile acid (TBA) levels [AOR $(95 \% \mathrm{Cl}) 1.014(1.009-1.020)$ ] and higher RDW [AOR $(95 \% \mathrm{Cl}) 1.582(1.261-1.986)]$. The result for area under the curve of 0.905 for TBA levels indicated this variable had high diagnostic performance for predicting DILI severity. Based on an area under the curve value of $0.855, \mathrm{RDW}$ also had superior diagnostic performance in prediction of DILI severity. This performance was not significantly different compared with TBA and was superior compared with other variables, which had area under values ranging from poor to failure (0.527-0.714).The risk for severe DILI was associated with drinking, dyslipidemia, higher TBA levels and RDW values. This study found that RDW and TBA levels were predictors of DILI severity in Chinese patients.

Although limited data exist on the incidence among the general population of drug-induced liver injury (DILI), it is an important public health problem because many medications are likely involved in the occurrence of liver injury ${ }^{1,2}$. Because DILI is a difficult disease to diagnose, the actual values for incidence are likely greater than reported. Diverse individual characteristics that contribute to specific host-drug interactions result in a variety of clinical presentations that complicate the diagnosis of $\mathrm{DILI}^{3,4}$. Some degree of repair to liver damage typically occurs when the drug or drugs are stopped. However, in a small percentage of patients, DILI can persist and progress after drug removal.

Variability in red blood cell (RBC) size can be measured using red blood cell distribution width (RDW). RDW measurement is typically included in complete blood cell counts ${ }^{5-7}$. Study results indicate that RDW can be used as an independent predictor of the prognosis of diseases including renal diseases, cardiovascular diseases, pulmonary hypertension, lung cancer, and sepsis ${ }^{8-14}$. Significant associations have been found between RDW and chronic liver disease severity (e.g., nonalcoholic fatty liver disease, alcoholic cirrhosis, and chronic hepatitis $\mathrm{B}(\mathrm{CHB}))^{15-17}$. Elevated RDW levels have been found in patients with autoimmune liver disease such as primary biliary cholangitis and autoimmune hepatitis ${ }^{18,19}$. DILI progression and severity is associated with inflammation and autoimmune reaction ${ }^{20}$. However, the association between RDW values and DILI severity is mostly unknown.

The objective of this study was to analyze risk factors for DILI severity in a Chinese population. We evaluated and compared the performance of noninvasive serum sample-associated markers (i.e., total bile acid (TBA) levels, RDW, RDW-to-platelet count (PLT) ratio (RPR), RDW-to-lymphocyte ratio (RLR), and aspartate aminotransferase-to-alanine aminotransferase ratio (AAR) when used to diagnosis disease severity in 164 Chinese patients with DILI.

\footnotetext{
${ }^{1}$ Department of Hepatology, The First Hospital of Jilin University, Jilin University, No. 71 Xinmin Street, Changchun 130021, China. ${ }^{2}$ Key Laboratory of Organ Regeneration and Transplantation of Ministry of Education, The First Hospital of Jilin University, Changchun 130061, People's Republic of China. ${ }^{3}$ Jilin Province Key Laboratory of Infectious Disease, Laboratory of Molecular Virology, Changchun 130021, China. ${ }^{\circledR}$ email: gpj@jlu.edu.cn
} 


\begin{tabular}{|l|c|c|}
\hline Variable & Total DILI (N= 164) & Normal range \\
\hline Male, N(\%) & $40(24.4)$ & \\
\hline Age (years) & $50.00(42.00,56.00)$ & \\
\hline Smoking, N(\%) & $24(14.6)$ & \\
\hline Drinking, N(\%) & $20(12.2)$ & \\
\hline Hypersensitivity history, N(\%) & $29(17.7)$ & \\
\hline Hypertension, N(\%) & $26(15.9)$ & \\
\hline Dyslipidemia, N(\%) & $59(36.0)$ & \\
\hline Diabetes, N(\%) & $14(8.5)$ & \\
\hline WBC count $\left(10^{9} / \mathrm{L}\right)$ & $4.98(3.94,6.47)$ & $3.50-9.50$ \\
\hline Neutrophil $\left(10^{9} / \mathrm{L}\right)$ & $2.74(1.96,3.59)$ & $1.80-6.30$ \\
\hline Lymphocyte $\left(10^{9} / \mathrm{L}\right)$ & $1.69(1.24,2.19)$ & $1.10-3.20$ \\
\hline Hemoglobin $(\mathrm{g} / \mathrm{L})$ & $128.00(120.00,140.00)$ & $115-150$ \\
\hline Platelet $\left(10^{9} / \mathrm{L}\right)$ & $201.00(164.00,253.00)$ & $125-350$ \\
\hline RDW $(\%)$ & $14.30(13.10,15.83)$ & $11.0-14.0$ \\
\hline MCV $(\mathrm{fL})$ & $89.90(86.40,93.30)$ & $82-100$ \\
\hline AST $(\mathrm{IU} / \mathrm{L})$ & $117.80(46.55,230.60)$ & $13.0-35.0$ \\
\hline ALT $(\mathrm{IU} / \mathrm{L})$ & $164.35(55.03,330.85)$ & $7.0-40.0$ \\
\hline TBIL $(\mu \mathrm{mol} / \mathrm{L})$ & $29.40(13.70,122.13)$ & $0.0-6.8$ \\
\hline ALP $(\mathrm{IU} / \mathrm{L})$ & $121.90(96.80,189.78)$ & $35.0-100.0$ \\
\hline GGT $(\mathrm{IU} / \mathrm{L})$ & $147.65(77.40,275.53)$ & $7.0-45.0$ \\
\hline Globulin $(\mathrm{g} / \mathrm{L})$ & $28.40(24.60,31.85)$ & $20.0-40.0$ \\
\hline TBA $(\mu \mathrm{mmol} / \mathrm{L})$ & $25.60(8.20,136.45)$ & $0-10$ \\
\hline PT $(\mathrm{s})$ & $11.45(10.80,12.40)$ & $9.0-13.0$ \\
\hline INR & $0.99(0.93,1.06)$ & $0.8-1.2$ \\
\hline & & \\
\hline
\end{tabular}

Table 1. Baseline characteristics of the study population. Continuous variables are expressed as median (25th, 75th percentiles) values. Categorical variables were displayed as numbers and percentages. WBC white blood cell, $R D W$ red blood cell distribution width, $M C V$ mean corpuscular volume, $A S T$ aspartate aminotransferase, $A L T$ alanine aminotransferase, TBIL total bilirubin, $A L P$ alkaline phosphatase, GGT gamma-glutamyltransferase, TBA total bile acid, $P T$ prothrombin time, INR international normalized ratio.

\begin{tabular}{|l|l|l|l|}
\hline Symptoms & No of cases (\%) & Laboratory results & No of cases (\%) \\
\hline Fatigue & $101(61.6)$ & AST elevation & $140(85.4)$ \\
\hline Abdominal distension & $53(32.3)$ & ALT elevation & $141(86.0)$ \\
\hline Jaundice & $69(42.1)$ & GGT elevation & $145(88.4)$ \\
\hline Nausea & $24(14.6)$ & ALP elevation & $118(72.0)$ \\
\hline Pruritus & $1(0.61)$ & TbiL elevation & $101(61.6)$ \\
\hline None & $20(12.2)$ & TBA elevation & $114(69.5)$ \\
\hline
\end{tabular}

Table 2. Clinical characteristics of 164 patients with drug-induced liver injury. AST aspartate aminotransferase, $A L T$ alanine aminotransferase, GGT gamma-glutamyl transferase, ALP alkaline phosphatase, TbiL total bilirubin, TBA alkaline phosphatase.

\section{Results}

Demographic and clinical characteristics. Patient demographic and clinical characteristics are presented in Tables 1 and 2. The study population consisted of $24.4 \%$ men. The median age was $50.0(42.0,56.0)$ years. The values for prevalence of hypertension and dyslipidemia were 15.9\% (26/164) and 36\% (59/164), respectively. The prevalence of diabetes mellitus and a history of hypersensitivity were $8.5 \%(14 / 164)$ and $17.7 \%$ (29/164), respectively.

Clinical characteristics of patients with DILI are shown in Table 2. The most prevalent clinical manifestations were fatigue (61.6\%) and jaundice (42.1\%). Abdominal distension and nausea accounted for 32.3\% and $14.6 \%$, respectively. A total of $12.2 \%$ of the patients had no symptoms or clinical signs.

Therapeutic classes and uses of drugs. The therapeutic classes of drugs used by participants in the DILI group are listed in Table 3. Of note, 79 DILI patients (48.2\%) used Chinese herbal medicines, 56 (34.1\%) used Western medicines, and 29 (17.7\%) used a combination of the two. These drugs are suspected to be the cause of liver injury in DILI patients. To further evaluate the indication of the herbal drugs, we subdivided the 79 patients 


\begin{tabular}{|l|l|l|}
\hline & No. of cases & Percentage \\
\hline Chinese herbal medicines & 79 & 48.2 \\
\hline Western medicines & 56 & 34.1 \\
\hline Both & 29 & 17.7 \\
\hline
\end{tabular}

Table 3. Therapeutic classes of drugs that caused liver injury in 164 Chinese patients.

\begin{tabular}{|l|l|}
\hline Cause & No of cases \\
\hline Dietary supplements & 30 \\
\hline Anti-inflammatory drugs & 12 \\
\hline Cardiovascular drugs & 10 \\
\hline Osteoarthropathy drugs & 7 \\
\hline Digestive system drugs & 7 \\
\hline Obstetric/gynecological drugs & 4 \\
\hline Rheumatism drugs & 3 \\
\hline Nervous/mental system drug & 3 \\
\hline Others & 9 \\
\hline
\end{tabular}

Table 4. Indications of drugs that caused liver injury in 79 Chinese DILI patients, induced by herbal medications. DILI drug-induced liver injury.

who used Chinese herbal medications into causal categories (Table 4). Dietary supplements, anti-inflammatory drugs, Cardiovascular drugs, Osteoarthropathy drugs, and digestive system drugs were the top five types of causal herbal drugs.

Univariate and multivariate analyses. We evaluated risk factors for severity of DILI in the 164 patients who had the condition (Table 5). The results for the univariate analysis indicated that drinking, dyslipidemia, TBA level, RDW level, RLR level, and AAR level were significantly different between patients with severe ( $\geq$ level 3 ) and mild-to-moderate (levels 0-2) disease. Sex, age, smoking, drinking, history of hypersensitivity, diabetes mellitus, history of liver disease, dyslipidemia, TBA, RDW, RPR, RLR, and AAR were included in the multivariate analysis. The AOR for patients with dyslipidemia was 3.926 (95\% CI 1.282-12.026; $P=0.017$ ), compared with patients with normal plasma lipid values. The AOR for patients who drank was 4.938 (95\% CI 1.088-22.412; $P=0.039$ ), compared with patients who did not drink. Compared with patients with lower TBA levels, patients with higher TBA levels had a higher risk for development of severe DILI (1.014 [95\% CI 1.009-1.020], $P<0.001)$. Patients with higher RDW values had a higher risk for development of severe DILI (1.582 [95\% CI 1.261-1.986], $P<0.001)$. We found no significant associations between levels of RPR, RLR, AAR, and DILI severity.

Diagnostic performance and thresholds of serum models for severity in DILI patients. The results for the estimation of AUROC values to predict severe DILI indicated that the performance of TBA $(\mathrm{AUROC}=0.905,95 \% \mathrm{CI} 0.860-0.950)$ and RDW (AUROC $=0.855,95 \%$ CI 0.793-0.917) were higher than RLR $(\mathrm{AUROC}=0.714,95 \% \mathrm{CI} 0.620-0.807)$, AAR $(\mathrm{AUROC}=0.633,95 \% \mathrm{CI} 0.537-0.728)$, and RPR $(\mathrm{AUROC}=0.527$, 95\% CI 0.419-0.635) (Table 6, Fig. 1).

Maximizing the sum of sensitivity and specificity, the optimal cut-off for TBA was 45.850 for diagnosis of severe DILI. The results indicated that the sensitivity was $100 \%$ and the specificity was $74.2 \%$ for diagnosis of severe DILI. The correct classified percentage was 79.3\%. The optimal cut-off for RDW was 14.650 for diagnosis of severe DILI. The sensitivity was $86.1 \%$ and the specificity was $73.4 \%$ for diagnosis of severe DILI. The correct classified percentage was $76.2 \%$.

\section{Discussion}

The most important findings of this study were that the results further supported the hypotheses that there was a positive correlation between TBA levels and DILI severity and that RDW predicted DILI severity. At a cutoff of 14.650, RDW predicted severe DILI with a sensitivity of $86.1 \%$ and a specificity of $73.4 \%$. The percent correctly classified could be as high as $76.2 \%$ with an AUROC of 0.855 . These diagnostic capabilities were similar to TBA and were higher than those for other non-invasive markers.

In hepatic diseases, such as viral hepatitis, fatty liver disease, and liver cirrhosis ${ }^{21,22}$, serum bile acid (BA) levels can increase from 10- to 100-fold ${ }^{21-25}$. Compared with serum bilirubin, BA measurement may be a more sensitive method to assess hepatic function ${ }^{23}$. Changes in serum BAs correlate with the severity of hepatic dysfunction. Horvatits et al.'s results indicated that TBA levels can be used as markers to stratify risk in patients with cirrhosis with respect to new onset of acute decompensation and acute-on-chronic liver failure ${ }^{21}$.

Numerous studies have examined associations between RDW levels and liver-related disease progression and outcomes. One large sample size cohort study found that RDW is predictive marker for advanced fibrosis in 


\begin{tabular}{|c|c|c|c|c|c|}
\hline Variables & Level 0-2 $(\mathrm{N}=128)$ & Level $\geq 3(\mathrm{~N}=36)$ & $P^{*}$ & AOR $(95 \% \mathrm{CI})^{*}$ & $P^{* *}$ \\
\hline \multicolumn{3}{|l|}{ Sex } & 0.157 & - & - \\
\hline Female, N(\%) & $100(78.1)$ & $24(66.7)$ & & & \\
\hline Male, N(\%) & $28(21.9)$ & $12(33.3)$ & & & \\
\hline \multicolumn{3}{|l|}{ Age } & 0.353 & - & - \\
\hline$<60$ & $108(84.4)$ & $28(77.8)$ & & & \\
\hline$\geq 60$ & $20(15.6)$ & $8(22.2)$ & & & \\
\hline \multicolumn{3}{|l|}{ Smoking } & 0.355 & - & - \\
\hline No, N(\%) & $111(86.7)$ & $29(80.6)$ & & & \\
\hline Yes, $\mathrm{N}(\%)$ & $17(13.3)$ & $7(19.4)$ & & & \\
\hline \multicolumn{3}{|l|}{ Drinking } & 0.017 & $4.938(1.088-22.412)$ & 0.039 \\
\hline No, N(\%) & $117(91.4)$ & $27(75.0)$ & & & \\
\hline Yes, $\mathrm{N}(\%)$ & $11(8.6)$ & $9(25.0)$ & & & \\
\hline \multicolumn{3}{|c|}{ Hypersensitivity history } & 0.096 & - & - \\
\hline No, N(\%) & $102(79.7)$ & $33(91.7)$ & & & \\
\hline Yes, N(\%) & $26(20.3)$ & $3(8.3)$ & & & \\
\hline \multicolumn{3}{|l|}{ Diabetes } & 0.737 & - & - \\
\hline No, $\mathrm{N}(\%)$ & $116(90.6)$ & $34(94.4)$ & & & \\
\hline Yes, $\mathrm{N}(\%)$ & $12(9.4)$ & $2(5.6)$ & & & \\
\hline \multicolumn{3}{|c|}{ History of liver disease } & 0.524 & - & - \\
\hline No, N(\%) & $99(77.3)$ & $26(72.2)$ & & & \\
\hline Yes, $\mathrm{N}(\%)$ & $29(22.7)$ & $10(27.8)$ & & & \\
\hline \multicolumn{3}{|l|}{ Dyslipidemia } & 0.006 & $3.926(1.282-12.026)$ & 0.017 \\
\hline No, N(\%) & $89(69.5)$ & $16(44.4)$ & & & \\
\hline Yes, $\mathrm{N}(\%)$ & $39(30.5)$ & $20(55.6)$ & & & \\
\hline \multicolumn{3}{|l|}{ INR } & 0.180 & & \\
\hline$<1.0$ & $73(57.0)$ & $16(44.4)$ & & & \\
\hline$\geqq 1.0$ & $55(43.0)$ & $20(55.6)$ & & & \\
\hline TBA & $14.75(7.40,56.95)$ & $185.60(132.75,271.85)$ & \begin{tabular}{c|c}
$<001$ \\
\end{tabular} & $1.014(1.009-1.020)$ & $<0.001$ \\
\hline RDW & $13.75(12.80,14.80)$ & $16.65(14.95,19.70)$ & $<0.001$ & $1.582(1.261-1.986)$ & $<0.001$ \\
\hline RPR & $0.07(0.05,0.09)$ & $0.07(0.06,0.09)$ & 0.617 & & \\
\hline RLR & $7.61(6.10,10.72)$ & $10.68(8.44,17.97)$ & $<0.001$ & & \\
\hline AAR & $0.71(0.50,0.96)$ & $0.91(0.69,1.15)$ & 0.015 & & \\
\hline
\end{tabular}

Table 5. Univariate and multivariate analyses of variables associated with severe drug-induced liver injury. Continuous variables are expressed as median (25th, 75th percentiles). Categorical variables were displayed as numbers and percentages. INR international normalized ratio, $R L R$ red blood cell distribution width to lymphocyte ratio, $R P R$ red blood cell distribution width to platelet ratio, $A A R$ aspartate aminotransferase to alanine aminotransferase ratio. ${ }^{\#} P$ value for univariate analysis. ${ }^{*}$ Adjusted for sex, age, smoking, drinking, allergic history, DM, hyperlipemia, history of liver disease, TBA, RDW, RPR, RLR, and AAR. ${ }^{*} P$ value for multivariate analysis.

patients with nonalcoholic fatty liver disease ${ }^{26}$. Fan et al. found that RDW is elevated in patients with $\mathrm{CHB}$ and that there is a positive correlation between these changes and $\mathrm{CHB}$ severity ${ }^{27}$. RDW is significantly increased in patients with HCC; it represents an easily measured marker of prognosis in these patients ${ }^{28,29}$.

Abnormalities including inflammation, erythrocyte fragmentation, oxidative stress, poor nutritional condition, and abnormality of erythropoietin function can cause significant variation in RDW ${ }^{5,30-34}$. Because these disorders and anemia correlate with liver disease severity, elevated RDW values might also be associated with the severity of liver disease. Inflammation results in impairment of erythrocyte maturation and entry of immature erythrocytes into the systemic circulation that results in elevated RDW values ${ }^{19,35}$. Study results suggest that inflammatory cytokines (e.g., tumor necrosis factor- $\alpha$, interleukin (IL)- $1 \beta$, and IL-6) inhibit iron metabolism and erythropoietin production. This process results in disorders of RBC synthesis and abnormal erythropoietin production $^{35-37}$. Impairment of the balance between oxidant and antioxidant defenses are characteristic of the oxidative stress that occurs in liver disease. Erythrocyte homeostasis and survival is strongly affected by oxidative stress, and low serum antioxidant concentrations are correlated with increased RDW levels ${ }^{38,39}$. Taken together, this suggests oxidative stress results in increased RDW levels in patients with liver disease. A liver disease-associated poor nutritional status (e.g., iron deficiency, folate deficiency, vitamin B12 deficiency) can also result in abnormal RBC production and increased RDW levels ${ }^{40}$. Patients with chronic liver disease can experience the common complication of portal hypertension. This condition can cause splenomegaly with an associated increase in the rate of RBC destruction and release of immature RBCs to the systemic circulation and consequent increase in $\mathrm{RDW}^{38,39}$. 


\begin{tabular}{|l|l|l|l|l|l|l|}
\hline Variables & AUROC & $\mathbf{( 9 5 \%} \mathbf{C I})$ & Cut-off & Se (\%) & Sp (\%) & Correct classified (\%) \\
\hline RPR & 0.527 & $0.419-0.635$ & 0.073 & 58.3 & 52.3 & 53.0 \\
\hline AAR & 0.633 & $0.537-0.728$ & 0.599 & 86.1 & 41.4 & 51.2 \\
\hline RLR & 0.714 & $0.620-0.807$ & 10.227 & 66.7 & 71.1 & 70.1 \\
\hline RDW & 0.855 & $0.793-0.917$ & 14.650 & 86.1 & 73.4 & 76.2 \\
\hline TBA & 0.905 & $0.860-0.950$ & 45.850 & 100 & 74.2 & 79.3 \\
\hline Comparison of AUROC & & & & & & \\
\hline TBA and RDW & $P>0.05$ & & & & & \\
\hline RDW and RLR & $P<0.05$ & & & & & \\
\hline RLR and AAR & $P>0.05$ & & & & & \\
\hline AAR and RPR & $P>0.05$ & & & & & \\
\hline RLR and RPR & $P<0.05$ & & & & & \\
\hline
\end{tabular}

Table 6. Diagnostic performance of serum models for severe drug-induced liver injury. The bold values were considered statistically significant $(P<0.05)$. Se, sensitivity; Sp, specificity. Cut-offs were established by maximizing the sum of sensitivity and specificity. AUROC area under the receiver operating characteristic curve, 95\% CI 95\% confidence interval.

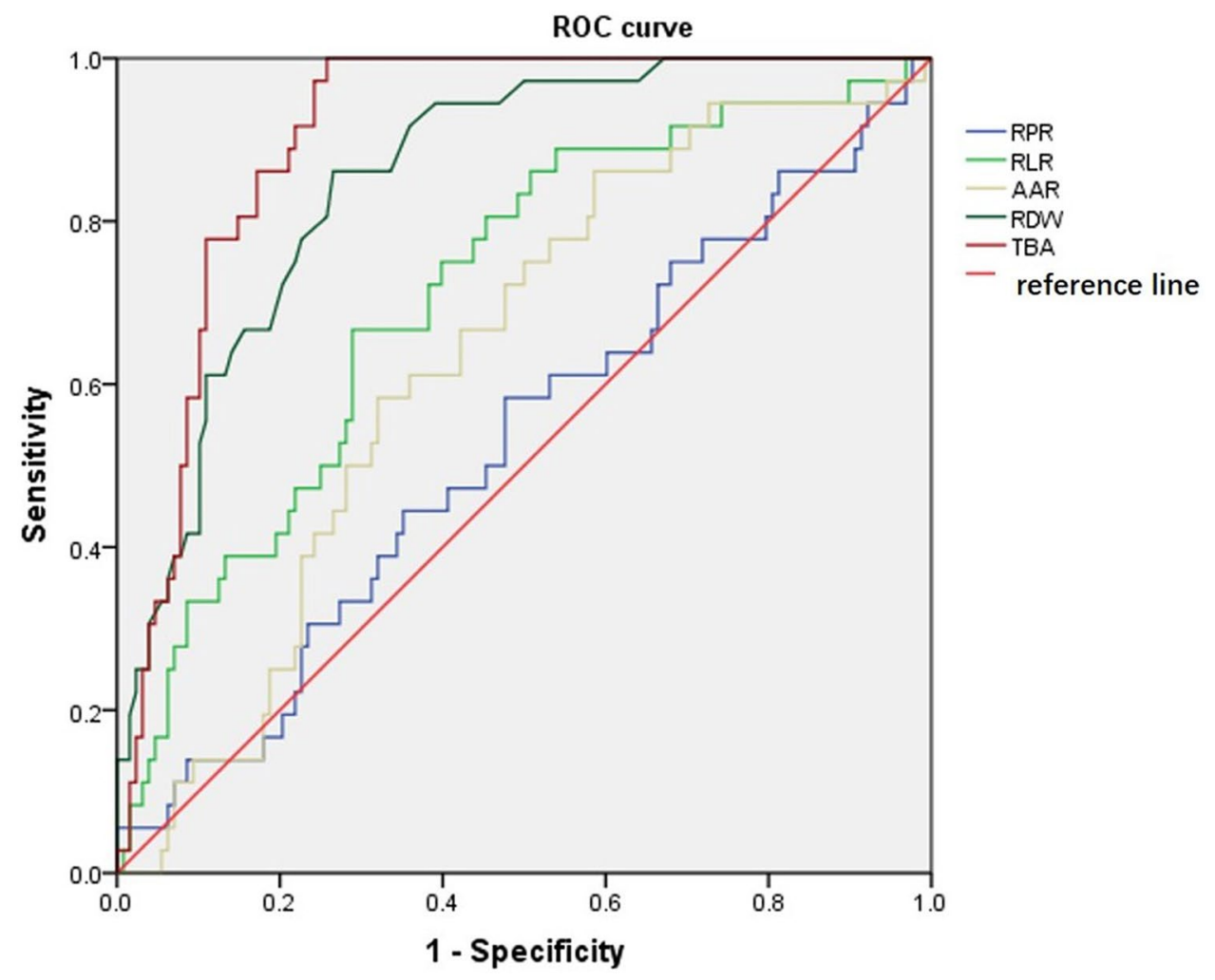

Figure 1. Receiver operating characteristic (ROC) curves of red blood cell distribution width (RDW), RDWto-platelet ratio (RPR), RDW-to-lymphocyte ratio (RLR), total bile acid (TBA), and aspartate transaminase-toalanine aminotransferase ratio (AAR) for diagnosis of severe DILI.

Our study did not find relationships between RPR, RLR, and severe DILI. One explanation for this result is that the patients were not further analyzed (because of the sample size) to determine whether they had liver cirrhosis, which would have influenced PLT levels. Innate/adaptive immune responses have key roles in the biological mechanisms of DILI ${ }^{41}$ and could also could affect blood cell levels. Consistent with the results of other studies, we found associations between dyslipidemia, drinking, and the severity of DILI ${ }^{2}$. The detailed mechanisms have been described in our previous study ${ }^{2,41}$.

There are some limitations to our study. First, the study's retrospective design might have caused selection bias that resulted in underestimated sensitivity and overestimated specificity values in the serum models ${ }^{42}$. Second, detailed information about lipid-lowering drugs and hypoglycemic agents was not available. More study is needed to understand associations between drugs used for metabolic disease and severe DILI. Third, the patients 
included in our study were not combined with other liver disease to tried to avoid factors which might influence RDW value, however we cannot avoid other system diseases because that might the reason patients use drugs. Fourth, because the study did not include a large sample size, subgroup analyses by acute and chronic DILI could not be performed. The number of cases was limited by the requirements to include patients with a diagnosis of DILI with liver biopsy and exclude patients without complete medical information.

In conclusion, risk for severe DILI was associated with drinking, dyslipidemia, higher TBA levels, and higher RDW. This study found that RDW and TBA levels may be valid for non-invasive, real-time monitoring of DILI severity in Chinese patients.

\section{Methods}

Patients. Data from 2097 patients who underwent standard laboratory tests and liver biopsies (The First Hospital of Jilin University, China) between January 2010 and December 2019 were assessed for inclusion in this retrospective study. Test and biopsy results indicated that 187 patients had a diagnosis of DILI. After excluding the data from 23 patients who had incomplete medical information, the data from 164 patients with complete medical histories and laboratory information were included in the analysis.

Subjects were excluded due to the following The exclusion criteria: adopted were (1) co-infection with human immunodeficiency virus or hepatitis B virus (HBV) or hepatitis C virus (HCV); (2) the evidence or history or other evidence of hepatitis (3) presence of other types of liver disease, such as (e.g., non-alcoholic fatty liver disease or, alcoholic liver disease, et al.

The Independent Institutional Review Board of The First Hospital of Jilin University approved the study protocol and the use of data from human subjects. All participants in the study signed informed consent.

Liver biopsy. Each ultrasound-guided percutaneous liver biopsy was performed using the Menghini technique. All liver samples were preserved in phosphate-buffered formalin and then paraffin-embedded and sectioned and stained for histology. Liver activity and fibrosis were scored using the Metavir system ${ }^{43}$. We excluded tissue sections with fewer than three portal tracts (i.e., as poor quality). The pathologists were blinded to all clinical data. If their conclusions were different, the slides were examined by a another experienced hepatopathologist in the UK who was blinded to all data.

Diagnosis of DILI. Dyslipidemia was defined using criteria from the 2015 Chinese Medical Association guidelines for DILI diagnosis and treatment ${ }^{44}$ : All patients's RUCAM score more and equal to 6 , and the presence of liver injury was finally diagnosed based on liver histology of percutaneous liver biopsies.

Diagnosis of DILI severity. The 2015 Chinese Medical Association guidelines for DILI diagnosis and treatment were used to classify severity into five categories (level 0: exposure to drug, but no liver injury; level 1: mild, typically reversible elevations of serum enzyme activities only, total bilirubin $(\mathrm{TBil})<2.5$ the upper limit of normal (ULN) value and international normalized ratio (INR) $<1.5$; level 2: more extensive injury with early impairment of liver function indicated by increases in alanine aminotransferase (ALT) and/or alkaline phosphatase (ALP), TBil $\geq 2.5$ ULN or INR $\geq 1.5$; level 3: serious clinical illness in conjunction with obvious jaundice and disabling symptoms (TBil $\geq 5 \mathrm{ULN}$ and/or INR $\geq 1.5$ ); level 4: increases in ALT and/or ALP, TBil $\geq 10$ ULN or TBil increases $\geq 17.1 \mu \mathrm{mol} / \mathrm{L}$ per day, INR $\geq 2.0$ or prothrombin activity $<40 \%$ in addition to secondary loss of other organ functions (e.g., encephalopathy or hepatorenal syndrome); level 5: liver required transplant or death of patient ${ }^{44}$.

Diagnosis of dyslipidemia. Dyslipidemia was defined using criteria from the National Cholesterol Education Program Adult Treatment Panel III (ATPIII), which were total cholesterol greater than $240 \mathrm{mg} / \mathrm{dL}$, HDL cholesterol less than $40 \mathrm{mg} / \mathrm{dL}$, LDL cholesterol greater than or equal to $160 \mathrm{mg} / \mathrm{dL}$, or triglycerides greater than or equal to $200 \mathrm{mg} / \mathrm{dL}^{45}$.

RDW-to-lymphocyte ratio, RDW-to-platelet count ratio, and aspartate aminotransferase-to-alanine aminotransferase ratio. RLR was calculated using RLR=RDW (\%)/lymphocyte $\left(10^{9} / \mathrm{L}\right)$, RPR was calculated using $\mathrm{RPR}=\mathrm{RDW}(\%) / \mathrm{PLT}\left(10^{9} / \mathrm{L}\right)$, and AAR was calculated using AAR $=\mathrm{AST}$ (IU/L)/ALT (IU/L).

Study variables. Demographic characteristics (e.g., age, sex, drinking, smoking) and variables associated with the clinical presentation (presence of hypertension, history of hypersensitivity, presence of dyslipidemia, type 2 diabetes) were included in this study.

At the time of liver biopsy, fasting blood samples were obtained for standard laboratory tests. Retrospective data on white blood cell (WBC), neutrophil, and lymphocyte counts, and on hemoglobin, RDW, platelet counts, mean corpuscular volume, serum aspartate aminotransferase (AST), ALT, gamma glutamyl transferase, ALP, TBil, TBA, and globulin levels were obtained from the patient's medical record.

The INR, prothrombin activity, and prothrombin time were also analyzed in the patients with DILI and were used to classify severity of liver injury.

Statistical analysis. The results for continuous variables were calculated as median, and 25th and 75th percentile values and analyzed using two-tailed independent sample t-tests. Categorical variables were summarized as numbers/percentages and analyzed using Chi-squared tests. To adjust for potential confounding effects, 
multivariate logistic regression analyses were performed, including adjusted odds ratios (AORs) and 95\% confidence intervals (CIs). We used SPSS software (version 13, SPSS Inc., Chicago, IL, USA) to perform the statistical analyses. All tests were two-tailed. We considered $P$ values $<0.05$ to be statistically significant.

Receiver operating characteristic (ROC) curves and the area under the ROC (AUROC) curve were used to evaluate and compare the accuracy of AAR, RPR, RLR, TBA levels, and RDW for the diagnosis of DILI severity. ROC curve analysis and Z-tests were used to compute and compare AUROCs, respectively (MedCalc Software bvba, version 16, Ostend, Belgium). Maximizing the sum of sensitivity and specificity or optimizing a specificity of at least 95\% were used to obtain cut-offs. All methods were carried out in accordance with relevant guidelines and regulations.

Received: 22 September 2020; Accepted: 16 December 2020

Published online: 12 January 2021

\section{References}

1. Bjornsson, E. S., Bergmann, O. M., Bjornsson, H. K., Kvaran, R. B. \& Olafsson, S. Incidence, presentation, and outcomes in patients with drug-induced liver injury in the general population of Iceland. Gastroenterology. 144(7), 1419-1425, 25.e1-25.e3; quiz e19-e20 https://doi.org/10.1053/j.gastro.2013.02.006 (2013).

2. Li, X., Wang, L., Li, D., Niu, J. \& Gao, P. Dyslipidemia is a risk factor for the incidence and severity of drug-induced liver injury (DILI): a retrospective population-based study in China. Med. Sci. Monit. 25, 3344-3353. https://doi.org/10.12659/msm.916687 (2019).

3. Lucena, M. I. et al. Phenotypic characterization of idiosyncratic drug-induced liver injury: the influence of age and sex. Hepatology 49(6), 2001-2009. https://doi.org/10.1002/hep.22895 (2009).

4. Kleiner, D. E. et al. Hepatic histological findings in suspected drug-induced liver injury: systematic evaluation and clinical associations. Hepatology 59(2), 661-670. https://doi.org/10.1002/hep.26709 (2014).

5. Lippi, G., Dipalo, M., Teti, L. \& Cervellin, G. Relationship between red blood cell distribution width and prognostic biomarkers in patients admitted to the emergency department with acute infections. Eur. J. Intern. Med. 24(2), e15-e16. https://doi.org/10.1016/j. ejim.2012.09.005 (2013).

6. Bessman, J. D., Hurley, E. L. \& Groves, M. R. Nondiscrete heterogeneity of human erythrocytes: comparison of Coulter-principle flow cytometry and Soret-hemoglobinometry image analysis. Cytometry 3(4), 292-295. https://doi.org/10.1002/cyto.990030411 (1983).

7. Salvagno, G. L., Sanchis-Gomar, F., Picanza, A. \& Lippi, G. Red blood cell distribution width: a simple parameter with multiple clinical applications. Crit. Rev. Clin. Lab. Sci. 52(2), 86-105. https://doi.org/10.3109/10408363.2014.992064 (2015).

8. Montagnana, M., Cervellin, G., Meschi, T. \& Lippi, G. The role of red blood cell distribution width in cardiovascular and thrombotic disorders. Clin. Chem. Lab. Med. 50(4), 635-641. https://doi.org/10.1515/cclm.2011.831 (2011).

9. Arbel, Y. et al. Red blood cell distribution width (RDW) and long-term survival in patients with ST elevation myocardial infarction. Thromb. Res. 134(5), 976-979. https://doi.org/10.1016/j.thromres.2014.08.016 (2014).

10. Yoon, H. E. et al. Progressive rise in red blood cell distribution width predicts mortality and cardiovascular events in end-stage renal disease patients. PLoS ONE 10(5), e0126272. https://doi.org/10.1371/journal.pone.0126272 (2015).

11. Jo, Y. H. et al. Red cell distribution width is a prognostic factor in severe sepsis and septic shock. Am. J. Emerg. Med. 31(3), 545-548. https://doi.org/10.1016/j.ajem.2012.10.017 (2013).

12. Lee, J. H. et al. Incremental predictive value of red cell distribution width for 12 -month clinical outcome after acute myocardial infarction. Clin. Cardiol. 36(6), 336-341. https://doi.org/10.1002/clc.22114 (2013).

13. Koma, Y. et al. Increased red blood cell distribution width associates with cancer stage and prognosis in patients with lung cancer. PLoS ONE 8(11), e80240. https://doi.org/10.1371/journal.pone.0080240 (2013).

14. Huang, C. F. et al. Baseline gamma-glutamyl transferase levels strongly correlate with hepatocellular carcinoma development in noncirrhotic patients with successful hepatitis C virus eradication. J. Hepatol. 61(1), 67-74. https://doi.org/10.1016/j.jhep.2014.02.022 (2014).

15. Milic, S., Mikolasevic, I., Radic, M., Hauser, G. \& Stimac, D. Clinical utility of red cell distribution width in alcoholic and nonalcoholic liver cirrhosis. Coll. Antropol. 35(Suppl 2), 335-338 (2011).

16. Lou, Y., Wang, M. \& Mao, W. Clinical usefulness of measuring red blood cell distribution width in patients with hepatitis B. PLoS ONE 7(5), e37644. https://doi.org/10.1371/journal.pone.0037644 (2012).

17. $\mathrm{Hu}, \mathrm{Z}$. et al. Red blood cell distribution width is a potential prognostic index for liver disease. Clin. Chem. Lab. Med. 51(7), 1403-1408. https://doi.org/10.1515/cclm-2012-0704 (2013).

18. Wang, H. et al. Red blood cell distribution width to platelet ratio is related to histologic severity of primary biliary cirrhosis. Medicine 95(11), e3114. https://doi.org/10.1097/md.0000000000003114 (2016).

19. Wang, H. et al. Red blood cell distribution width for predicting significant liver inflammation in patients with autoimmune hepatitis. Eur. J. Gastroenterol. Hepatol. 31(12), 1527-1532. https://doi.org/10.1097/meg.0000000000001447 (2019).

20. de Boer, Y. S. et al. Features of autoimmune hepatitis in patients with drug-induced liver injury. Clin. Gastroenterol. Hepatol. 15(1), 103-12.e2. https://doi.org/10.1016/j.cgh.2016.05.043 (2017).

21. Horvatits, T. et al. Serum bile acids as marker for acute decompensation and acute-on-chronic liver failure in patients with noncholestatic cirrhosis. Liver Int. 37(2), 224-231. https://doi.org/10.1111/liv.13201 (2017).

22. Li, T. \& Chiang, J. Y. Bile acids as metabolic regulators. Curr. Opin. Gastroenterol. 31(2), 159-165. https://doi.org/10.1097/ mog.0000000000000156 (2015).

23. Korman, M. G., Hofmann, A. F. \& Summerskill, W. H. Assessment of activity in chronic active liver disease. Serum bile acids compared with conventional tests and histology. N. Engl. J. Med. 290(25), 1399-1402. https://doi.org/10.1056/nejm19740620290 2503 (1974).

24. Murphy, G. M., Ross, A. \& Billing, B. H. Serum bile acids in primary biliary cirrhosis. Gut 13(3), 201-206. https://doi.org/10.1136/ gut.13.3.201 (1972).

25. Neale, G., Lewis, B., Weaver, V. \& Panveliwalla, D. Serum bile acids in liver disease. Gut 12(2), 145-152. https://doi.org/10.1136/ gut.12.2.145 (1971).

26. Kim, H. M. et al. Elevated red cell distribution width is associated with advanced fibrosis in NAFLD. Clin. Mol. Hepatol. 19(3), 258-265. https://doi.org/10.3350/cmh.2013.19.3.258 (2013).

27. Fan, X. et al. Association of red blood cell distribution width with severity of hepatitis B virus-related liver diseases. Clin. Chim. Acta. 482, 155-160. https://doi:10.1016/j.cca.2018.04.002 (2018).

28. Goyal, H. \& Hu, Z. D. Prognostic value of red blood cell distribution width in hepatocellular carcinoma. Ann. Transl. Med. 5(13), 271. https://doi.org/10.21037/atm.2017.06.30 (2017).

29. Wei, T. T. et al. Elevated red blood cell distribution width is associated with liver function tests in patients with primary hepatocellular carcinoma. Clin. Hemorheol. Microcirc. 64(2), 149-155. https://doi.org/10.3233/ch-162053 (2016). 
30. Lippi, G. \& Plebani, M. Red blood cell distribution width (RDW) and human pathology. One size fits all. Clin. Chem. Lab. Med. 52(9), 1247-1249. https://doi.org/10.1515/cclm-2014-0585 (2014).

31. Lee, H. et al. Elevated red blood cell distribution width as a simple prognostic factor in patients with symptomatic multiple myeloma. Biomed. Res. Int. 2014, 145619. https://doi.org/10.1155/2014/145619 (2014).

32. Tekce, H., Kin Tekce, B., Aktas, G., Tanrisev, M. \& Sit, M. The evaluation of red cell distribution width in chronic hemodialysis patients. Int. J. Nephrol. 2014, 754370. https://doi.org/10.1155/2014/754370 (2014).

33. Kim, C. H. et al. An increase in red blood cell distribution width from baseline predicts mortality in patients with severe sepsis or septic shock. Crit. Care (London, England) 17(6), R282. https://doi.org/10.1186/cc13145 (2013).

34. Senol, K., Saylam, B., Kocaay, F. \& Tez, M. Red cell distribution width as a predictor of mortality in acute pancreatitis. Am. J. Emerg. Med. 31(4), 687-689. https://doi.org/10.1016/j.ajem.2012.12.015 (2013).

35. Lippi, G. et al. Relation between red blood cell distribution width and inflammatory biomarkers in a large cohort of unselected outpatients. Arch. Pathol. Lab. Med. 133(4), 628-632. https://doi.org/10.1043/1543-2165-133.4.628 (2009).

36. Krintus, M., Kozinski, M., Kubica, J. \& Sypniewska, G. Critical appraisal of inflammatory markers in cardiovascular risk stratification. Crit. Rev. Clin. Lab. Sci. 51(5), 263-279. https://doi.org/10.3109/10408363.2014.913549 (2014).

37. Allen, L. A. et al. Validation and potential mechanisms of red cell distribution width as a prognostic marker in heart failure. J. Card. Fail. 16(3), 230-238. https://doi.org/10.1016/j.cardfail.2009.11.003 (2010).

38. Semba, R. D. et al. Serum antioxidants and inflammation predict red cell distribution width in older women: the Women's Health and Aging Study I. Clin. Nutr. (Edinburgh, Scotland). 29(5), 600-604. https://doi.org/10.1016/j.clnu.2010.03.001 (2010).

39. Friedman, J. S. et al. SOD2-deficiency anemia: protein oxidation and altered protein expression reveal targets of damage, stress response, and antioxidant responsiveness. Blood 104(8), 2565-2573. https://doi.org/10.1182/blood-2003-11-3858 (2004).

40. Mantovani, A., Allavena, P., Sica, A. \& Balkwill, F. Cancer-related inflammation. Nature 454(7203), 436-444. https://doi. org/10.1038/nature07205 (2008).

41. Li, X., Gao, P. \& Niu, J. Metabolic comorbidities and risk of development and severity of drug-induced liver injury. Biomed. Res. Int. 2019, 8764093. https://doi.org/10.1155/2019/8764093 (2019).

42. Choi, B. C. Sensitivity and specificity of a single diagnostic test in the presence of work-up bias. J. Clin. Epidemiol. 45(6), 581-586 (1992).

43. Bedossa, P. \& Poynard, T. An algorithm for the grading of activity in chronic hepatitis C. The METAVIR Cooperative Study Group. Hepatology 24(2), 289-293. https://doi.org/10.1002/hep.510240201 (1996).

44. God-iliiCM, A. Chinese guideline for diagnosis and treatment of DILI. J. Clin. Hepatol. 31(11), 1752-1769. https://doi.org/10.3969/j. issn.1001-5256.2015.11.002 (2015).

45. Executive Summary of The Third report of the National Cholesterol Education Program (NCEP). Expert panel on detection, evaluation, and treatment of high blood cholesterol in adults (Adult Treatment Panel III). JAMA 285(19), 2486-2497 (2001).

\section{Author contributions}

X.L.: Acquisition of data and administrative, technical, and material support, statistical analysis, drafting of the manuscript. H.X.: Acquisition of data and administrative. P.G.: Study concept and design, study supervision and critical revision of the manuscript for important intellectual content. All authors reviewed the manuscript.

\section{Competing interests}

The authors declare no competing interests.

\section{Additional information}

Correspondence and requests for materials should be addressed to P.G.

Reprints and permissions information is available at www.nature.com/reprints.

Publisher's note Springer Nature remains neutral with regard to jurisdictional claims in published maps and institutional affiliations.

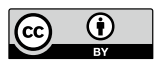

Open Access This article is licensed under a Creative Commons Attribution 4.0 International License, which permits use, sharing, adaptation, distribution and reproduction in any medium or format, as long as you give appropriate credit to the original author(s) and the source, provide a link to the Creative Commons licence, and indicate if changes were made. The images or other third party material in this article are included in the article's Creative Commons licence, unless indicated otherwise in a credit line to the material. If material is not included in the article's Creative Commons licence and your intended use is not permitted by statutory regulation or exceeds the permitted use, you will need to obtain permission directly from the copyright holder. To view a copy of this licence, visit http://creativecommons.org/licenses/by/4.0/.

(C) The Author(s) 2021 